\title{
CONTABILIDADE
}




\section{ASPECTOS DA PRODUÇÃO CIENTÍFICA SOBRE CONTROLE GERENCIAL}

ASPECTS OF SCIENTIFIC PRODUCTION REGARDING MANAGEMENT CONTROL

Cleyton de Oliveira Ritta

Fundação Universidade Regional de Blumenau

Carlos Eduardo Facin Lavarda

Universidade Federal de Santa Catarina

\author{
Data de submissão: 09 set. 20|6. Data de aprovação: \\ 07 abr. 2017. Sistema de avaliação: Double blind review. \\ Universidade FUMEC / FACE. Prof. Dr. Henrique Cordeiro \\ Martins. Prof. Dr. Cid Gonçalves Filho.
}

\section{RESUMO}

O objetivo geral da pesquisa é avaliar aspectos da produção científica sobre controle gerencial na perspectiva da contabilidade gerencial nos periódicos internacionais da base de dados da Web of Science, disponíveis até 2014. A pesquisa caracteriza-se como descritiva com abordagem quantitativa por meio de estudo bibliométrico. Os procedimentos de coleta resultaram em 32 artigos disponíveis em oito periódicos. Os resultados da pesquisa mostraram que: maioria dos artigos foi do tipo empírico e de natureza exploratória. $O$ foco das pesquisas tem como temas a funcionalidade de artefatos contábeis e da estrutura do sistema de controle gerencial.A teoria da Contingência foi a principal base teórica do constructo dos artigos. Conclui-se que as pesquisas sobre controle gerencial possuem amplo campo de assuntos para futuras pesquisas. Os fenômenos organizacionais contemporâneos e as novas estruturas organizacionais demandam por estudos exploratórios para compreender a função do sistema de controle gerencial na condução dos negócios.

\section{PALAVRAS-CHAVE}

Controle Gerencial. Contabilidade Gerencial. Pesquisa Científica. Bibliometria. 


\section{ABSTRACT}

The overall objective of this research is to evaluate aspects of the scientific production regarding management control from the perspective of management accounting in international journals in the Web of Science database, available until 2014. The research is characterized as descriptive with a quantitative approach conducted via bibliometric study. The collection procedures resulted in 32 items available in 8 journals. The survey results have shown that: most articles were empirical and exploratory articles. The focus of the research was the functionality of accounting devices and the structure of the management control system. Contingency theory was the main theoretical basis of the construct of the articles. It can be concluded that research on management control has a vast field of topics for future research. Contemporary organizational phenomena and new organizational structures demand exploratory studies to understand the function of the management control system in conducting business.

\section{KEYWORDS}

Management Control. Management Accounting. Scientific Research. Bibliometrics.

\section{INTRODUÇÃO}

As mudanças do ambiente econômico e organizacional exigem das organizações mecanismos de gestão para o controle das atividades. $O$ controle gerencial é um mecanismo de gestão que tem o papel de monitorar o desempenho das operações e fornecer informações para a tomada de decisão. Segundo Anthony e Govindarajan (2006), a função do controle gerencial é assegurar que as estratégias organizacionais sejam obedecidas pelos colaboradores para atingir os objetivos estabelecidos.

$\mathrm{Na}$ configuração dos sistemas de controle gerencial, segundo Merchant e Van Der Stede (2007), as organizações devem estipular as dimensões de desempenho desejadas e não desejadas, identificar as métricas para a mensuração do desempenho e criar incentivos para que os colaboradores persigam as metas estabelecidas. Com essas premissas de estrutura de controle gerencial, as organizações estimulam a congruência entre os objetivos dos colaboradores e organizacionais.

A contabilidade gerencial fornece as principais métricas para a configuração de sistemas de controle gerencial, com a função de auxiliar no processo de tomada de decisão de gestores; além de contribuir para otimização do desempenho organizacional. De acordo com Atkinson, Banker, Kaplan et al. (2000), a contabilidade gerencial disponibiliza informações para o controle das atividades, avaliação de desempenho organizacional, planejamento de estratégias e tomada de decisão.

A complexidade do ambiente de negócios exerce influência na estrutura de sistema de controle gerencial e nas práticas de contabilidade gerencial nas organizações. Esse ambiente desperta o interesse de pes- 
quisadores da área de gestão em verificar e compreender o impacto da complexidade do ambiente de negócios na condução das atividades organizacionais. Segundo Shields (1997), as pesquisas científicas são motivadas pela necessidade de compreensão das mudanças do ambiente interno e externo que afetam as organizações.

De acordo com esse contexto, surge a seguinte pergunta de pesquisa: qual o perfil da produção científica sobre controle gerencial na perspectiva da contabilidade gerencial nos periódicos internacionais da base de dados da Web of Science? O objetivo geral da pesquisa é investigar aspectos da produção científica sobre controle gerencial na perspectiva da contabilidade gerencial nos periódicos internacionais da base de dados da Web of Science, disponíveis até $\mathrm{o}$ ano de 20I4. Para atingir o objetivo geral, estipulam-se os seguintes objetivos específicos: a) verificar características dos artigos e autores e b) identificar características dos assuntos abordados nos artigos.

Estudos sobre o perfil da produção científica internacional sobre controle gerencial e contabilidade gerencial ganham relevância na literatura contábil com os autores Shields (1997), Hesford, Lee, Van Der Stede et al. (2007), Faro e Silva (2008), Nascimento, Ribeiro e Junqueira (2008), Cunha, Klann e Lavarda (20l3), Beuren e Nascimento (20l4), Brizolla, Chiarello e Lavarda (20।4). Esses estudos revelam vários aspectos da produção científica sobre contabilidade gerencial.

O principal ponto de convergência dos estudos foi que as pesquisas evidenciaram que o tema mais discutido foi o controle gerencial com foco na avaliação de desempenho organizacional. As pesquisas identificaram aspectos da estrutura de controle gerencial, práticas contábeis utilizadas por organizações, influência dos estágios de mudança organizacional nos controles gerenciais e estrutura metodológica dos trabalhos.

Diante dos resultados dos estudos anteriores, percebe-se que o tema controle gerencial sobressai em relação aos demais que envolvem a contabilidade gerencial. Por isso, a presente pesquisa investiga de modo específico outras características da produção científica sobre controle gerencial, no intuito de contribuir com o aprofundamento das discussões e identificar novos caminhos de pesquisa sobre o tema.

\section{REFERENCIAL TEÓRICO}

Esta seção discorre sobre controle gerencial, contabilidade gerencial e estudos anteriores relacionados ao tema.

\section{Controle Gerencial e Contabilidade Gerencial}

O controle gerencial é um processo de atividades de gestão utilizado pela alta administração para mobilizar os colaboradores no cumprimento das metas e estratégias organizacionais de curto e longo prazo (ANTHONY; GOVINDARAJAN, 2006; LUNKES; SCHNORRENBERGER, 2009).

O controle gerencial serve para coordenar, monitorar e avaliar o desempenho organizacional. Por isso, é a principal base para o desenvolvimento de ações estratégicas e para tomada de decisão. Segundo Mahama (2006), o controle gerencial compreende arranjos organizacionais que servem para assegurar o alcance das metas e corrigir possíveis desvios de resultados. Otley e Berry (1980) observam que os controles devem viabilizar a realização das estratégias, a coordenação e integração das atividades organizacionais e possibilitar 
a adaptação da organização às mudanças internas e externas do ambiente em que está inserida.

Segundo Flamholtz (1979), o controle gerencial influencia o comportamento dos colaboradores, de modo a incentivá-los a alcançar os objetivos organizacionais. A motivação é um elemento fundamental para que os indivíduos alinhem os objetivos pessoais com os objetivos organizacionais (HENRI, 2006; MAHAMA, 2006).

$O$ conjunto de elementos de controle gerencial caracteriza-se como sistema de controle gerencial. Para Simons (1995), o sistema de controle gerencial configura-se como rotinas e procedimentos organizacionais que geram informações para que os gestores as utilizem para manter ou alterar os padrões de atividades e para o alcance das estratégias. Atkinson, Banker, Kaplan et al. (2000) expõem que os principais objetivos do sistema de controle gerencial são: a) auxiliar no planejamento do futuro organizacional, b) monitorar o ambiente organizacional, c) medir e registrar as atividades, d) motivar os colaboradores e e) avaliar o desempenho da empresa e dos colaboradores.

A estrutura do sistema de controle gerencial depende das características das organizações e do ambiente em que atuam. Para Gomes e Amat (1995), o sistema de controle resulta na interação entre técnicas formais e informais de gestão e aspectos socioculturais como comportamento dos indivíduos, cultura organizacional, instituições sociais e políticas. Portanto, as organizações devem promover estruturas organizacionais flexíveis que facilitem a interação entre diferentes indivíduos, artefatos de controle gerencial e ambiente organizacional, para que ocorra a otimização de recursos e o alcance das estratégias planejadas.
Segundo Aguiar e Frezatti (2007), uma estrutura adequada de sistema de controle gerencial é composta: a) por técnicas tradicionais e contemporâneas de gestão que coletam e registram dados para a geração de informações sobre o desempenho das atividades; b) por informações gerenciais amplamente aceitas e que representam fatos apurados de modo sistêmico e tempestivo e c) pela satisfação dos usuários no uso das informações para a tomada de decisão e nos possíveis benefícios gerados.

Dentre os elementos que compõe o sistema de controle gerencial, tem-se a contabilidade gerencial como principal fornecedora de informações sobre os processos organizacionais. Segundo Atkinson, Banker, Kaplan et al. (2000), o objetivo da contabilidade gerencial é auxiliar os gestores na identificação, mensuração, avaliação e divulgação de informações sobre os eventos econômicos da organização para a tomada de decisão. Guerreiro, Frezatti e Casado (2006, p. 10) ressaltam que a missão da contabilidade gerencial é "prover informações adequadas para que os tomadores de decisões maximizem o resultado econômico de suas decisões".

A contabilidade gerencial atende os interesses de proprietários, gestores e demais colaboradores por meio de informações operacionais, financeiras e não financeiras que os ajudam a compreender e aperfeiçoar os processos organizacionais e que dão suporte ao planejamento e o desenvolvimento de vantagens competitivas (OTLEY, 1994; KAPLAN; NORTON, 2000).

A contabilidade gerencial contempla diversas práticas organizacionais denominadas de artefatos gerenciais. Conforme Frezatti, Rocha, Nascimento et al. (2009), artefatos são conceitos, ferramentas, mo- 
delos ou sistemas utilizados para ajudar na execução de tarefas ou efetuar determinada função. Os artefatos da contabilidade gerencial podem ser classificados como tradicionais - Custeio por Absorção, Orçamento Tradicional, Custeio Variável - e contemporâneos - Custeio ABC (Activity Based Costing), Custeio Meta (Target Costing), Balanced Scorecard (BSC), Economic Value Added (EVA), Market Value Added (MVA), Activity Based Management (ABM), entre outros.

O surgimento de artefatos contemporâneos sinaliza a evolução da contabilidade gerencial nas últimas décadas. Segundo International Federation of Accountants [IFAC] (I998), as mudanças da contabilidade gerencial ocorreram para atender as necessidades do ambiente organizacional. Até a década de 1950, os artefatos auxiliavam na determinação dos custos e nos controles financeiros. Com o passar dos tempos, outros artefatos foram necessários para atender necessidades de planejamento, controle operacional, redução de desperdício e geração de valor. Os artefatos contemporâneos possibilitam a maximização de recursos, o monitoramento de estratégias e o desenvolvimento de vantagens competitivas.

Chenhall (2003) esclarece as características que distinguem as concepções de contabilidade gerencial, sistema de contabilidade gerencial e sistema de controle gerencial. A contabilidade gerencial contempla um conjunto de práticas organizacionais como orçamento, sistemas de custeio, indicadores econômico-financeiros e controle operacional, entre outros que auxiliam na condução dos negócios; enquanto o sistema de contabilidade gerencial é a utilização sistemática dos artefatos da contabilidade gerencial para alcançar os objeti- vos estratégicos. Por sua vez, o sistema de controle gerencial é mais amplo e engloba o sistema de contabilidade gerencial e outros controles gerenciais como controle de processo, de produção, de qualidade, de pessoas e de informação para apoio à tomada de decisão e alcance das estratégias organizacionais.

Portanto, o controle gerencial e a contabilidade gerencial constituem-se como principais bases gerenciais para a geração de informação e tomada de decisão. Esses elementos evoluíram para acompanhar as mudanças do ambiente organizacional e econômico, por meio de novos artefatos gerenciais, no intuito de obter informações mais adequadas, tempestivas e relevantes para a gestão das organizações.

\section{Estudos Anteriores}

Estudos anteriores sobre o perfil da produção científica sobre controle gerencial ou contabilidade gerencial no contexto internacional são destaques na literatura contábil. $O$ pesquisador Shields (1997) investigou aspectos da produção científica em contabilidade gerencial em periódicos internacionais no período entre 1990 a 1996. Os resultados mostraram que o tema mais pesquisado foi sistemas de controle gerencial com ênfase em políticas de incentivos e avaliação de desempenho.

Para o pesquisador, as pesquisas em contabilidade gerencial foram motivadas pelas mudanças de ambiente interno e externo das organizações e, por isso, é preciso novos estudos para compreender os seguintes aspectos: a) fatores inibidores e propulsores da mudança nos sistemas de controle gerencial e contabilidade gerencial, b) práticas contábeis em organizações com estruturas horizontais, c) relações en- 
tre contabilidade e estratégia corporativa, d) informação contábil e desenvolvimento tecnológico virtual.

Hesford, Lee, Van Der Stede et al. (2007) analisaram as publicações sobre contabilidade gerencial em periódicos internacionais no período de 1981 a 2000. A coleta totalizou 916 artigos nos seguintes periódicos: Accounting, Organizations and Society (AOS), Behavioral Research in Accounting (BRIA), Contemporary Accounting Research (CAR), Journal of Accounting and Economics $(J A E)$, Journal of Accounting Literature (JAL), Journal of Accounting Research (JAR), Journal of Management Accounting Research (JMAR), Management Accounting Research (MAR), Review of Accounting Studies (RAS) e The Accounting Review (TAR).

Os resultados mostraram que os artigos de contabilidade gerencial representaram $28 \%$ de toda a publicação no período. Os periódicos com maior quantidade de artigos sobre o tema foram AOS e MAR, que juntos representam mais da metade dos artigos. A maioria dos artigos foi classificada: a) na categoria controle, b) do tipo teórico e c) pertencente às disciplinas de economia e sociologia. Os métodos de pesquisa mais empregados foram pesquisa teórica, analítica e survey.

Faro e Silva (2008) investigaram a produção acadêmica na área de contabilidade gerencial no âmbito internacional entre os anos de 1997 a 2007. A pesquisa foi realizada em cinco periódicos internacionais considerados como os mais influentes na área contábil: Accounting Organization and Society (AOS), Contemporary Accounting Research (CAR), Journal of Accounting and Economics (JAE), Journal of Accounting Research (JAR), The Accounting Review (AR).

A pesquisa totalizou em 239 artigos que abordaram o tema contabilidade gerencial. Os resultados evidenciaram que o foco das pesquisas está voltado ao assunto sistemas de controle gerencial relacionado às políticas de incentivos e de avaliação de desempenho. Por outro lado, os assuntos orçamento, controle financeiro e custos apresentam declínio de publicações. Além disso, de modo pulverizado, constataramse tendências de crescimento de pesquisas nos assuntos balanced scorecard, inovação, comportamento, estratégia e estrutura organizacional.

Nascimento, Ribeiro e Junqueira (2008) descreveram as características das pesquisas com abordagem comportamental no âmbito da contabilidade gerencial em periódicos internacionais entre os anos de 1997 a 2007.A pesquisa contemplou os periódicos que continham no título a palavra "accounting" disponíveis no portal CAPES. Tal processo resultou em 49 periódicos e uma amostra de 125 artigos. Os resultados evidenciaram que os periódicos mais relevantes foram Behavioral Accounting Research (BAR) e Accounting, Organizations and Society (AOS).

Os assuntos mais frequentes nas publicações foram a) julgamento e tomada de decisão em contabilidade gerencial e b) avaliação de desempenho organizacional e incentivos/remuneração. As estratégias metodológicas mais empregadas foram experimento e survey com abordagem positivista. A maioria dos artigos foi produzida com apenas um autor. $\mathrm{O}$ autor mais representativo na rede científica foi Chee Choow com maior grau de centralidade e de intermediação.

Cunha, Klann e Lavarda (20I3) analisaram o perfil da produção científica sobre ciclo de vida organizacional com aborda- 
gem ao controle gerencial em periódicos internacionais. Para seleção dos periódicos, os autores consideraram aqueles que continham no título a palavra "accounting". Esse procedimento resultou em 67 periódicos com 39 artigos. Os resultados mostraram que o periódico com maior número de publicações foi Management Accounting Research (MAR), com três artigos publicados. A pesquisadora Hanna Silvola possui o maior número de publicação sobre o tema.

Os artigos investigaram as relações entre os estágios do ciclo de vida organizacional e a utilidade e a estrutura dos sistemas de controle gerencial. Os estudos quantitativos predominaram, com utilização de questionários para coleta de dados e aplicação de análises quantitativas. Como sugestão para futuras pesquisas, os autores recomendaram a continuidade e ampliação da pesquisa, visto a importância do tema e pela pouca quantidade de artigos encontrados.

Beuren e Nascimento (2014) investigaram o perfil dos artigos de Contabilidade Gerencial publicados na Accounting, Organizations and Society (AOS). A amostra da pesquisa contemplou 186 artigos no período de 2005 a 2009. Os resultados demonstraram que os artigos de natureza empírica com abordagem quantitativa foram predominantes. As pesquisas foram realizadas principalmente em empresas privadas. Quanto aos aspectos metodológicos, as pesquisas foram realizadas por dois ou mais autores.

Os autores mais prolíficos foram Christopher S. Chapman, da Universidade de Oxford do Reino Unido, David J. Cooper, da Universidade do Estado da Flórida, e Dean Neu, da Universidade de Calgary do Canadá. As teorias de base mais utilizadas para fundamentar os estudos foram Teoria da Contingência, Teoria Ator-Rede e Teoria Institucional. A maioria dos artigos foi classificada nas disciplinas de Sociologia, Economia, Produção e Gerenciamento Organizacional. O tema e o assunto mais discutidos foram controle gerencial e mensuração/avaliação de desempenho, respectivamente.

Brizolla, Chiarello e Lavarda (20/4) identificaram aspectos sociométricos e bibliométricos das pesquisas sobre controladoria e contabilidade gerencial nos artigos publicados em periódicos internacionais. A base para coleta foi a Science Direct para estudos publicados entre os anos de 1993 a 2012 . A coleta resultou em 19 periódicos internacionais e 95 artigos relacionados ao tema. Os resultados apontaram que o periódico que mais publicou artigos sobre o tema foi o Accounting, Organizations and Society (AOS).

Os artigos foram produzidos em maior número por dupla de pesquisadores, a estrutura das redes é fragmentada e os autores possuem pouca publicação sobre o tema. $\bigcirc$ artigo com o maior número de citações foi "Assessing Empirical Research in Managerial Accounting: a value -based management perspective", de Ittner e Larcker (200I). Os assuntos mais discutidos foram desempenho organizacional, práticas de contabilidade gerencial e contabilidade como ferramenta para o controle gerencial.

De acordo com os estudos anteriores, percebe-se que o tema controle gerencial predomina nas pesquisas sobre contabilidade gerencial. Em linha com os estudos anteriores, a presente pesquisa dá continuidade ao assunto, porém investiga o tema de modo mais específico, conforme as propo- 
sições de Berry, Coad, Harris et al. (2009). Segundo esses autores, é preciso verificar as características das pesquisas sobre controle gerencial, principalmente quanto aos temas discutidos e assuntos emergentes que necessitam de pesquisas futuras.

\section{METODOLOGIA DA PESQUISA}

Para investigar o perfil da produção científica sobre controle gerencial na perspectiva da contabilidade gerencial nos periódicos da base de dados da Web of Science, realiza-se uma pesquisa: a) de natureza descritiva, uma vez que se descrevem as características dos trabalhos publicados sobre o tema; b) com abordagem quantitativa, uma vez que se categoriza e mensura aspectos da produção científica; c) do tipo bibliométrica, nos periódicos disponíveis em meio eletrônico da área de gestão, e d) por meio de análise documental de dados secundários (MARTINS;THEÓPHILO, 2009).

O foco da pesquisa são os periódicos disponíveis no site da base de dados Web of Science no dia 18 de março de 20I5.A referida base se justifica por contemplar periódicos científicos relevantes e multidisciplinares de todo o mundo. Para a coleta dos artigos, utilizou-se a função Pesquisa Básica no site da base de dados, considerando: a) termo-chave - "management control", b) domínio de pesquisa - social sciences, c) área de pesquisa - business economics e d) tipo de documento - article e review.

O resultado dessa busca totalizou em 827 artigos. Na sequência, foram eliminados 469 artigos, pois os textos não estavam disponíveis de modo integral. Em seguida, excluíram-se seis artigos de periódicos com títulos não alusivos à área de gestão e oito revisões de livros, resultando em uma amostra de 344 artigos. Posteriormente, por meio de leitura, selecionaram-se 123 artigos que continham o termo-chave no título, resumo e palavras-chave. Por fim, por meio de leitura completa dos artigos, selecionaram-se 32 artigos como amostra final, uma vez que apresentavam em seu constructo o conceito, objetivos ou funções da contabilidade gerencial.

Para a coleta dos dados foi elaborado um formulário de pesquisa em planilhas do Microsoft Excel ${ }^{\circledR}$ que continha os seguintes elementos: a) características dos autores e artigos - nome, instituição, ano de publicação, nome do periódico, fator de impacto 20 I4/JCR ${ }^{\circledR}$ do periódico; b) aspectos metodológicos - tipo do estudo, natureza do objetivo de pesquisa, abordagem do problema de pesquisa, natureza do estudo, técnica de coleta de dados e técnica de análise de dados; c) referências - autores, título da obra, ano de publicação, tipo de fonte, nome do periódico e fator de impacto $20 / 4 / J C R^{\circledR}$ do periódico - e d) assuntos abordados - temas de pesquisa, teorias organizacionais utilizadas e sugestões para futuras pesquisas.

Em relação aos aspectos metodológicos dos artigos, consideraram-se as principais classificações conceituadas por Martins e Theóphilo (2009): a) tipo do estudo - teórico e empírico; b) natureza do objetivo de pesquisa - exploratório, descritivo, explicativo, mista; c) abordagem do problema de pesquisa - qualitativo, quantitativo e quali-quantitativo; d) natureza do estudo - bibliográfico, estudo de caso, multicasos, levantamento, mista;e) técnica de coleta de dados - entrevista, questionário, documentos, observação, mista e f) técnica de análise de dados - conteúdo, documental, quantitativa, mista. Para classificação metodológica, utilizaram-se as 
declarações disponíveis nos artigos, caso não declarado considerou-se a percepção dos autores da pesquisa.

No que concerne à classificação dos temas de pesquisa dos artigos, aplicou-se a técnica de análise de contéudo (BARDIN, 1979) para identificação de categorias e de frequências de temas recorrentes nas publicações. O Quadro I apresenta as características dos temas de pesquisa utilizados.

$\mathrm{Na}$ elaboração das categorias de temas de pesquisa, utilizou-se de forma adaptada a classificação proposta por Berry, Coad, Harris et al. (2009) em artigo que aborda uma revisão de literatura sobre os temas emergentes na área de controle gerencial, com ênfase na contabilidade gerencial. Além disso, consideraram-se as obras de Anthony e Govindarajan (2006) e Frezatti, Rocha, Nascimento et al. (2009) que tratam de controle gerencial.

\section{Descrição e Análise dos Resultados}

A descrição e análise dos resultados estão dispostas de acordo com os objetivos específicos propostos: a) características dos artigos e autores e b) características dos assuntos abordados nos artigos.

\section{Características dos Artigos \\ e Autores}

Os procedimentos de coleta resultaram em 32 artigos sobre controle gerencial (management control) até o ano de 20l4. A Tabela I mostra a distribuição temporal da produção científica.

No período, a maioria dos artigos é: a) empírica $(75,00 \%)$, b) exploratória $(62,50 \%)$; c) qualitativa $(53,13 \%)$; d) tipo estudo de caso/multicasos e bibliográficos (56,25\%); e) com técnica de coleta mista para dados - entrevista, documentos e observação (7I,88\%) - e f) e com técnica de

\section{QUADRO 1 - Temas de Pesquisa em Controle Gerencial Relacionados à Contabilidade Gerencial}

\begin{tabular}{|l|l|}
\hline Temas & Características \\
\hline Artefatos Contábeis e Controle Gerencial & $\begin{array}{l}\text { Configuração de artefatos da contabilidade gerencial para o controle gerencial no } \\
\text { que concerne à gestão, desenvolvimento de estratégias e avaliação de desempenho } \\
\text { organizacional. Exemplos: Balanced Scorecard, Orçamento, Sistemas de Custeio, } \\
\text { Sistemas de Gestão de Produção, Sistemas de Gestão de Informação, entre outros. }\end{array}$ \\
\hline Estrutura do Sistema de Controle Gerencial & $\begin{array}{l}\text { Utilidade e funcionalidade do sistema de controle gerencial, considerando objetivos } \\
\text { e estratégias organizacionais, medidas de controle, incentivo aos colaboradores, } \\
\text { fluxo de informações e a estrutura organizacional. }\end{array}$ \\
\hline $\begin{array}{l}\text { Aspectos Socioculturais relacionados ao } \\
\text { Sistema de Controle Gerencial }\end{array}$ & $\begin{array}{l}\text { Aspectos contextuais, culturais, históricos, sociais, políticos e comportamentais que } \\
\text { envolvem e influenciam os colaboradores o sistema de controle gerencial. }\end{array}$ \\
\hline $\begin{array}{l}\text { Abordagens teóricas e metodológicas sobre } \\
\text { o Sistema de Controle Gerencial }\end{array}$ & $\begin{array}{l}\text { Produção de conhecimento científico sobre sistema de controle gerencial e } \\
\text { contabilidade gerencial. }\end{array}$ \\
\hline Fonte: Adaptado de Anthony e Govindarajan (2006), Berry, Coad, Harris et al. (2009) e Frezatti, Rocha, Nascimento et al. (2009).
\end{tabular}

TABELA 1 - Distribuição Temporal da Produção Científica

\begin{tabular}{c|c|c|c}
\hline Décadas & Anos (Qtde.) & Qtde. & Freq. \% \\
\hline 1990 & $1987(1), 1991(1), 1995(2), 1997(1), 1999(1)$ & 6 & 18,75 \\
\hline 2000 & $2003(1), 2004(1), 2005(2), 2007(1), 2008(7), 2009(3)$ & 15 & 46,88 \\
\hline 2010 & $2010(1), 2011(2), 2012(1), 2013(3), 2014(4)$ & 11 & 34,37 \\
\hline \multicolumn{2}{|c|}{ Total Geral } & 32 & 100,00 \\
\hline
\end{tabular}

Fonte: Dados da pesquisa. 
análise de dados mista - conteúdo e documental (59,38\%).

Esses estudos têm por foco a compreensão da funcionalidade de artefatos contábeis e da estrutura do sistema de controle gerencial em organizações complexas e de grande porte, como hospitais, indústrias, multinacionais e bancos. Essas organizações ganham relevância nas pesquisas sobre controle gerencial, pois demandam por uma estrutura de sistema de controle sofisticada para dar suporte à gestão em um ambiente organizacional dinâmico e com várias unidades de negócios.

A Tabela 2 apresenta os autores mais produtivos sobre controle gerencial (management control) e a instituição a que estão vinculados.

Os 32 artigos foram produzidos por 64 autores que acumularam uma quantidade de 69 autorias. A pesquisadora Margaret A. Abernethy é a autora mais produtiva, com a quantidade de cinco artigos, seguida por Peter Brownell, com dois artigos. Observase que os demais autores não evidenciados produziram apenas um artigo.

Margaret A. Abernethy atua na Universidade de Melbourne na Austrália e desenvolve pesquisas na área de contabilidade gerencial, governança corporativa, estrutura de sistemas de compensação e contabilidade em instituições hospitalares. Em seus artigos, discute a influência dos controles contábeis e não contábeis no comportamento dos gestores, na estrutura do siste- ma de controle gerencial, no desempenho das organizações e no desenvolvimento de estratégias de mudança organizacional.

Dos cinco artigos produzidos pela autora, dois apresentam quantidade significativa de citações na base Web of Science, são eles: a) 104 citações - The impact of manufacturing flexibility on management control system design com coautoria de Anne M. Lillis e publicado no periódico Accounting, Organizations and Society (AOS) em 1995, e b) 103 citações - The role of budgets in organizations facing strategic change com coautoria de Peter Brownell e publicado no periódico Accounting, Organizations and Society (AOS) em 1999.

Dos 32 artigos, 17 (53, I3\%) foram publicados na Accounting, Organizations and Society (AOS) e nove $(28,13 \%)$ na Management Accounting Research (MAR). Esses periódicos são considerados relevantes na área contábil com fatores de impacto de I,672 e 2, I 25, respectivamente, segundo a métrica do Journal Citation Reports ${ }^{\circledR}$ (JCR) nos últimos dois anos.

Esse resultado corrobora com as pesquisas de Hesford, Lee, Van Der Stede et al. (2007), Nascimento, Ribeiro e Junqueira (2008) e Brizolla, Chiarello e Lavarda (20I4), que constataram que o periódico AOS contempla a maior parcela de artigos sobre contabilidade gerencial. Hesford, Lee, Van Der Stede et al. (2007) e Cunha, Klann e Lavarda (20I3) também perceberam uma quantidade relevante

TABELA 2 - Autores mais Produtivos

\begin{tabular}{c|l|c|c|c}
\hline Posição & \multicolumn{1}{|c|}{ Nome } & Instituição & Qtde. & Freq. \% \\
\hline $1^{\circ}$ & Margaret A. Abernethy & University of Melbourne & 5 & 7,25 \\
\hline $2^{\circ}$ & Peter Brownell & University of Melbourne & 2 & 2,90 \\
\hline \multicolumn{2}{|c|}{ Total } & & 7 & 10,15 \\
\hline
\end{tabular}

Fonte: Dados da pesquisa. 
de artigos sobre controle gerencial no periódico MAR.

Os 32 artigos totalizaram 2.79 I referências que foram classificadas em artigos, livros, teses/dissertação e outros documentos. A Tabela 3 apresenta a distribuição por cada tipo de fonte de pesquisa.

Os artigos (72,23\%) ganham a preferência dos pesquisadores por se configurarem como fontes mais dinâmicas e que abordam discussões atuais sobre fenômenos organizacionais. Além disso, os artigos ficam disponíveis em meio eletrônico, o que facilita acesso aos textos. Por sua vez, os livros $(25,62 \%)$ caracterizam-se como obras clássicas que despertaram os assuntos de pesquisa e que, portanto, são as fontes iniciais de estudo.

A Tabela 4 exibe os artigos mais referenciados.

A obra de Ouchi (1979) trata dos fa- tores mercado, estrutura burocrática e grupo de indivíduos que afetam a avaliação e o controle organizacional. A obra de Chenhall (2003) mostra uma revisão de literatura sobre sistemas de controle gerencial com abordagem da teoria da Contingência.A obra de Abernethy e Lillis (1995) identifica o impacto do processo de produção flexível na estrutura do sistema de controle gerencial.A obra de Bruns e Waterhouse (1975) investiga a influência do orçamento no comportamento humano e na estrutura organizacional.

Essas obras mostram a preocupação em compreender como os artefatos gerenciais e fatores ambientais internos e externos impactam configuração do sistema de controle gerencial e, consequentemente, na estrutura das organizações. Os estudos revelam que novos fenômenos organizacionais e novas formas de estrutura organizacional

TABELA 3 - Tipos de Fontes

\begin{tabular}{lc|c|c}
\hline \multicolumn{1}{c|}{ Tipos } & Qtde. & Freq. \% \\
\hline Artigos & 2.016 & 72,23 \\
\hline Livros & 715 & 25,62 \\
\hline Teses/Dissertações & 12 & 0,43 \\
\hline Outros & 48 & 1,72 \\
\hline & Total & 2.791 & 100,00 \\
\hline
\end{tabular}

Fonte: Dados da pesquisa.

TABELA 4 - Artigos mais Referenciados

\begin{tabular}{|c|c|c|c|c|}
\hline Autor & Título & Revista & Qtde. & Freq. \% \\
\hline Ouchi, W. G. (1979) & $\begin{array}{l}\text { A conceptual framework for the design of } \\
\text { organizational control mechanisms }\end{array}$ & $\begin{array}{l}\text { Management } \\
\text { Science }\end{array}$ & 11 & 0,39 \\
\hline Chenhall, R. H. (2003) & $\begin{array}{l}\text { Management control systems design within its } \\
\text { organizational context: finding from contingency- } \\
\text { based research and directions for the future }\end{array}$ & $\begin{array}{l}\text { Accounting, } \\
\text { Organizations } \\
\text { and Society }\end{array}$ & 9 & 0,32 \\
\hline $\begin{array}{l}\text { Abernethy, M. A.; Lillis, A. M. } \\
\text { (1995) }\end{array}$ & $\begin{array}{l}\text { The impact of manufacturing fexibility on } \\
\text { management control system design }\end{array}$ & $\begin{array}{l}\text { Accounting, } \\
\text { Organizations } \\
\text { and Society }\end{array}$ & 8 & 0,29 \\
\hline $\begin{array}{l}\text { Bruns, W. J.; Waterhouse, J. } \\
\text { H. (1975) }\end{array}$ & Budgetary control and organization structure & $\begin{array}{l}\text { Journal of } \\
\text { Accounting } \\
\text { Research }\end{array}$ & 8 & 0,29 \\
\hline \multicolumn{3}{|c|}{ Total } & 36 & 1,29 \\
\hline
\end{tabular}

Fonte: Dados da pesquisa. 
são determinantes na utilidade e funcionalidade do sistema de controle gerencial.

A Tabela 5 evidencia os livros mais referenciados.

Simons (1995) observa que o sistema de controle gerencial é utilizado para monitorar as atividades organizacionais e o comportamento dos colaboradores. A estrutura do sistema de controle é composta por quatro níveis ou alavancas, que são: sistemas de controle diagnósticos, sistemas de crenças, sistemas de limites e sistemas de controle interativos.

Mintzberg (1979) explica o funcionamento das organizações por meio de mecanismos de controle de processos, de resultados e de comportamento dos indivíduos para se conseguir parâmetros que definem as rotinas de negócios. A congruência desses mecanismos é exercida por meio da liderança gerencial para coordenar os indivíduos na execução das atividades.

Galbraith (1973) descreve o processo de gestão das organizações e mostra como a estrutura organizacional pode influenciar o desempenho. $O$ autor observa que para atender a complexidade organizacional e as incertezas do ambiente as organizações demandam por maiores quantidades de informações na gestão dos negócios.

Kaplan e Norton (1996) mostram como o balanced scorecard auxilia na transformação das estratégias em ação para alavancar o desempenho organizacional no curto e longo prazo. As quatro perspectivas do BSC (financeira, clientes, processos internos e aprendizado/crescimento) oferecem um equilíbrio de interesses organizacionais com o objetivo de assegurar a perpetuidade dos negócios.

As principais referências de artigos e livros revelam que as obras são literaturas da década de 90 . Isso evidencia que o assunto controle gerencial continua relevante para compreensão do funcionamento das organizações e do desempenho organizacional. Além disso, configuram-se como obras seminais que fundamentam estudos recentes.

A Tabela 6 apresenta os periódicos mais referenciados.

TABELA 5 - Livros mais Referenciados

\begin{tabular}{|c|c|c|c|}
\hline Autor & Título & Qtde. & Freq. \% \\
\hline Simons, R. (1995) & $\begin{array}{l}\text { Levers of control: how managers use innovative control systems } \\
\text { to drive strategic renewal }\end{array}$ & 10 & 0,36 \\
\hline Mintzberg, H. (1979) & The structuring of organizations & 8 & 0,29 \\
\hline Galbraith, J. R. (1973) & Designing complex organizations & 7 & 0,25 \\
\hline $\begin{array}{l}\text { Kaplan, R. S.; Norton, D. P. } \\
\text { (1996) }\end{array}$ & The balanced scorecard: translating strategy into action & 7 & 0,25 \\
\hline \multicolumn{2}{|r|}{ Total } & 32 & 1,15 \\
\hline
\end{tabular}

Fonte: Dados da pesquisa

TABELA 6 - Periódicos mais Referenciados

\begin{tabular}{l|c|c|c}
\hline \multicolumn{1}{c|}{ Títulos } & Fator de Impacto & Qtde. & Freq. \% \\
\hline Accounting, Organizations and Society & 1,672 & 428 & 15,34 \\
\hline Management Accounting Research & 2,125 & 143 & 5,12 \\
\hline Accounting Review & 2,267 & 94 & 3,37 \\
\hline \multicolumn{1}{c|}{ Total } & 665 & 23,83 \\
\hline
\end{tabular}

Fonte: Dados da pesquisa. 
A Accounting, Organizations and Society tem interesse em pesquisas que abordem relações entre contabilidade e comportamento humano, estrutura organizacional e processos, mudança do ambiente social e político das organizações.

A Management Accounting Research tem interesse em pesquisas da área da contabilidade gerencial que promovam contribuições para a consolidação dos temas de pesquisa e implicações práticas para gestão das organizações.

A Accounting Review tem interesse em pesquisas em contabilidade que apresente significativa contribuição nas áreas de sistemas de informação contábil, auditoria, contabilidade financeira, contabilidade gerencial e mais áreas correlatas.

Dentre os periódicos que possuem no título o termo "account", os três periódicos mais citados ocupam as principais posições no ranking de Fator de Impacto $20 \mid 4 / J C^{\circledR}$, na seguinte ordem: $3^{\mathrm{a}}$ posição - Accounting Review, $4^{\mathrm{a}}$ posição - Management Accounting Research e $5^{\mathrm{a}}$ posição - Accounting, Organizations and Society. Portanto, caracterizamse como periódicos científicos relevantes no cenário contábil mundial. Cabe mencionar que Journal of Accounting and Economics ocupa a $\mathrm{I}^{\mathrm{a}}$ posição com fator de impacto de 2,724, seguido do Journal of Accounting Research na $2^{\mathrm{a}}$ posição com fator de impacto de 2,384. Nesses dois periódicos não foram encontrados artigos conforme os procedimentos de pesquisa.

\section{Características dos Assuntos Abordados nos Artigos}

A Tabela 7 mostra a classificação dos artigos de acordo com os temas de pesquisa em controle gerencial.

No tema Artefatos Contábeis e Controle Gerencial, os artigos tratam da influência dos artefatos da contabilidade gerencial sobre o sistema de controle gerencial e como são utilizados para o desenvolvimento de estratégias, avaliação de desempenho, aprendizagem organizacional e atendimento às obrigações legais. Os artefatos gerenciais objetos de estudos foram orçamento, controle de produção (defeito zero/qualidade total), preço de transferência, balanced scorecard (BSC), indicadores de desempenho e enterprise resource planning systems (ERP).

No tema Estrutura do Sistema de Controle Gerencial, os artigos abordam aspectos da estrutura organizacional que impactam na configuração do sistema de controle gerencial. Os aspectos discutidos foram

TABELA 7 - Temas em Controle Gerencial na Perspectiva da Contabilidade Gerencial

\begin{tabular}{|c|c|c|c|}
\hline Temas & Características & Qtde. & Freq. \% \\
\hline Artefatos Contábeis e Controle Gerencial & $\begin{array}{l}\text { Configuração de artefatos da contabilidade gerencial para o } \\
\text { controle gerencial. }\end{array}$ & 9 & 28,13 \\
\hline $\begin{array}{l}\text { Estrutura do Sistema de Controle } \\
\text { Gerencial }\end{array}$ & Utilidade e funcionalidade do sistema de controle gerencial. & 9 & 28,13 \\
\hline $\begin{array}{l}\text { Abordagens teóricas e metodológicas } \\
\text { sobre o Sistema de Controle Gerencial }\end{array}$ & $\begin{array}{l}\text { Produção de conhecimento científico sobre sistema de } \\
\text { controle gerencial. }\end{array}$ & 8 & 25,00 \\
\hline $\begin{array}{l}\text { Aspectos Socioculturais relacionados aos } \\
\text { Sistemas de Controle Gerencial }\end{array}$ & $\begin{array}{l}\text { Aspectos contextuais, culturais, históricos, sociais, políticos } \\
\text { e comportamentais que envolvem o sistema de controle } \\
\text { gerencial. }\end{array}$ & 6 & 18,75 \\
\hline \multicolumn{2}{|r|}{ Total } & 32 & 100,00 \\
\hline
\end{tabular}

Fonte: Dados da pesquisa. 
controles contábeis e não contábeis, tipos de estratégias organizacionais (liderança em custo, diferenciação), sistemas de controle em organizações híbridas, controles interorganizacionais, práticas gerenciais institucionalizadas, inovação e estruturas interdepartamentais.

No tema Abordagens Teóricas e Metodológicas sobre Sistema de Controle Gerencial, os artigos realizaram revisão de estudos sobre controle gerencial com o objetivo de identificar novas possibilidades de pesquisa. Entre as possibilidades de futuras pesquisas destacam-se os assuntos: controle gerencial em interfirmas, controle gerencial e variáveis contingenciais emergentes, controle gerencial e comportamento dos colaboradores.

No tema Aspectos Socioculturais e Sistema de Controle Gerencial, os artigos versam sobre as relações comportamentais entre os colaboradores e a estrutura do sistema de controle gerencial. As relações investigadas foram a utilidade do sistema de controle para controlar comportamento, o efeito do estilo de liderança no sistema de controle, a percepção de colaboradores quanto aos objetivos do sistema de controle e os impactos no desempenho gerencial de colaboradores.

A Tabela 8 evidencia as teorias organizacionais mais citadas para fundamentar os artigos.

Dos 32 artigos, 27 (84,38\%) utilizaram as teorias organizacionais em seu constructo, com destaque para as teorias: Contingência $(38,25 \%)$,Agência $(20,59 \%)$ e Ator-Rede (II,76\%).

A teoria da Contingência é empregada para explicar que os sistemas de controle gerencial auxiliam na formulação e implantação de estratégias para enfrentar os fatores ambientais internos e externos. As variáveis contingenciais influenciam o uso dos artefatos gerenciais e a estruturação dos sistemas de controle. Logo, em um ambiente complexo e com muitas incertezas, as organizações possuem sistemas de gestão específicos para atender as demandas de negócios e as alterações do ambiente.

Segundo Otley (1980), a teoria da Contingência menciona que não existe um modelo de gestão universal que pode ser aplicado de forma igual para todas as organizações. No estudo de Beuren e Nascimento (20I4), a teoria da Contingência também predominou nos estudos sobre contabilidade gerencial.

A teoria da Agência é utilizada para explicar que os sistemas de controle gerencial disponibilizam informações sobre o desempenho organizacional para verificar a congruência entre os resultados planejados e alcançados. Os proprietários necessitam alinhar seus interesses aos dos agentes para que atendam as demandas organizacionais e, assim, consigam minimizar o risco das atividades e assegurar a continuidade do negócio no longo prazo.

Segundo Jensen e Meckling (1976), é na

TABELA 8 - Teorias Organizacionais

\begin{tabular}{l|c|c}
\hline \multicolumn{1}{c|}{ Teorias } & Qtde. & Freq. \% \\
\hline Teoria da Contingência & 14 & 43,75 \\
\hline Teoria da Agência & 5 & 15,63 \\
\hline Teoria do Ator-Rede & 5 & 15,63 \\
\hline Total & 24 & 75,01 \\
\hline
\end{tabular}

Fonte: Dados da pesquisa. 
relação principal-agente que surgem conflitos de interesses. Portanto, os sistemas de controle gerencial fornecem indicadores de desempenho para políticas de incentivos, no intuito de minimizar conflitos e motivar os colaboradores na execução das estratégias organizacionais estabelecidas pelos proprietários.

A teoria Ator-Rede é utilizada para explicar a interação entre colaboradores e artefatos ou sistemas de controle gerencial na construção da realidade organizacional. Os estudos focam em como os colaboradores impactam na estrutura dos sistemas de controle gerencial e vice-versa, com o objetivo de encontrar elementos relevantes no comportamento social.

Segundo Latour (2005), o ambiente social é constituído por elementos que possuem significados e que podem influenciar a rede de atores humanos e não humanos, principalmente quanto à aceitação ou rejeição de relacionamento. No estudo de Beuren e Nascimento (20।4), a teoria Ator-Rede também se destacou em estudos sobre contabilidade gerencial.

Constata-se que nas pesquisas sobre sistemas de controle gerencial as abordagens teóricas foram amplas e mostram a necessidade de análises multidisciplinares para a compreensão dos fenômenos organizacionais. As organizações são influenciadas por inúmeros fatores, como ambientais, com- portamentais e sociais. Por isso, a utilização de múltiplas abordagens teóricas revela diferentes aspectos da realidade organizacional que afetam a estrutura das entidades e desempenho dos colaboradores.

O Quadro 2 apresenta sugestões de assuntos para futuras pesquisas relacionadas ao sistema de controle gerencial.

De modo geral, os estudos apontam diversos assuntos para evolução das pesquisas em controle gerencial. Cabe destacar que os assuntos para futuras pesquisas estão relacionados ao ambiente contemporâneo em que as organizações estão inseridas e que, por isso, demandam por artefatos gerenciais e estruturas organizacionais complexos para a condução dos negócios. Observa-se que tais assuntos estão vinculados, principalmente, aos pressupostos da teoria da contingência, devido à maior representatividade dos estudos com essa abordagem na amostra de pesquisa.

Além disso, outra influência na tendência de assuntos futuros pode estar relacionada à concentração de estudos publicados nos periódicos Accounting, Organizations and Society (AOS) e Management Accounting Research (MAR), que possuem um forte interesse em temas que tratam da relação entre práticas de contabilidade gerencial e estrutura organizacional.

Esse resultado converge com as observações de Shields (1997), Faro e Silva

\section{QUADRO 2 - Assuntos para Futuras Pesquisas em Controle Gerencial}

\begin{tabular}{|l|l|}
\hline Foco & Assuntos de pesquisa \\
\hline Fenômeno organizacional & $\begin{array}{l}\text { aprendizagem organizacional, estratégia, preço de transferência, fatores contingenciais } \\
\text { contemporâneos, aspectos comportamentais e psicológicos dos colaboradores, } \\
\text { comportamento antiético dos colaboradores, desempenho organizacional, controles } \\
\text { interorganizacionais, relações e processos sociais, relações de poder, hierarquia } \\
\text { organizacional, inovação organizacional, múltiplos fatores contingenciais, ciclo de vida } \\
\text { organizacional, princípio de controlabilidade , software de gestão de informação (ERP), } \\
\text { novos artefatos gerenciais. }\end{array}$ \\
\hline Estrutura organizacional & complexa, multinacional, híbrida, joint venture, arranjos interorganizacionais. \\
\hline
\end{tabular}

Fonte: Dados da pesquisa. 
(2008) e Cunha, Klann e Lavarda (20/3) em relação às indicações de futuras pesquisas sobre controle gerencial. Shields (1997) menciona os seguintes assuntos: estratégia corporativa, tecnologia virtual e estrutura organizacional. Faro e Silva (2008) sugerem assuntos como inovação, comportamento, estratégia e estrutura organizacional. Cunha, Klann e Lavarda (20/3) indicam a investigação da relação entre ciclo de vida organizacional e estrutura de controle gerencial.

Os estudos empíricos da amostra indicam a continuidade das pesquisas por meio de replicação da metodologia de pesquisa para validação dos instrumentos de coleta, confirmação de hipóteses, identificação de outras variáveis ou fatores que impactam o fenômeno organizacional. Nos aspectos metodológicos, recomendam a ampliação da amostra, aplicação dos instrumentos de pesquisa em contextos diferenciados, utilização de outras métricas e métodos de pesquisa. Essas ações têm por objetivo contribuir para a consolidação e generalização de resultados.

Os estudos teóricos, por sua vez, sugerem o desenvolvimento de novas proposições teóricas que considerem a atualidade do ambiente de negócios e as novas formas de estrutura organizacional. Os estudos recomendam criação de métricas para mensuração de variáveis e a realização de testes empíricos para validar modelos teóricos.

Outra recomendação encontrada nos estudos é a necessidade de pesquisas mais amplas do tipo transversal e longitudinal para possibilitar o desenvolvimento de uma estrutura teórica consistente para explicar os fenômenos organizacionais que afetam ou são afetados pelo sistema de controle
gerencial.As pesquisas transversais possibilitam a verificação da diversidade de características do sistema de controle gerencial nas organizações e auxiliam na compreensão da relevância de semelhanças e diferenças nas estruturas de controle. Enquanto as pesquisas longitudinais permitem a compreensão dos efeitos das práticas organizacionais na estrutura do sistema de controle e como os colaboradores interagem com as mudanças do ambiente organizacional.

\section{CONSIDERAÇÕES FINAIS}

A pesquisa teve por objetivo geral investigar aspectos da produção científica sobre controle gerencial na perspectiva da contabilidade gerencial nos periódicos internacionais da base de dados da Web of Science, até $\circ$ ano de 2014. Os procedimentos de coleta resultaram em 32 artigos disponíveis em oito periódicos.

Os resultados, em relação às características dos artigos e autores, evidenciaram que a maioria dos artigos foi do tipo empírico (75\%) e de natureza exploratória (62,50\%). A pesquisadora Margaret A. Abernethy foi a autora mais produtiva, com cinco publicações $(7,25 \%)$. O periódico que mais publicou sobre o tema foi Accounting, Organizations and Society, com 17 artigos (53,I3\%). O principal tipo de fonte de pesquisa foi artigos $(72,23 \%)$.

$O$ artigo mais referenciado foi $A$ conceptual framework for the design of organizational control mechanisms, de Ouchi (1979), com I I citações $(0,39 \%)$. O livro mais referenciado foi Levers of control: how managers use innovative control systems to drive strategic renewal, de Simons (1995), com 10 citações (0,36\%). O periódico mais referenciado foi Accounting, Organizations and Society, com 428 citações (I5,34\%). 
Os resultados, quanto às características dos assuntos abordados, evidenciaram que o foco das pesquisas tem como temas a funcionalidade de artefatos contábeis $(28,13 \%)$ e a estrutura do sistema de controle gerencial $(28,13 \%)$. A teoria da Contingência foi a principal base teórica para explicar os fenômenos organizacionais relacionados à estrutura do sistema de controle gerencial (43,75\%). Os artigos sugeriram a investigação de assuntos emergentes que afetam o sistema de controle gerencial como, por exemplo: estratégia organizacional, inovação, aspectos socioculturais, alianças organizacionais, softwares de gestão, estrutura organizacional complexa e mudanças no ambiente interno e externo das organizações.

De acordo com os resultados, concluise que as pesquisas sobre controle gerencial possuem amplo campo de assuntos para futuras pesquisas. Os fenômenos organizacionais contemporâneos e as novas estruturas organizacionais demandam por estudos exploratórios para compreender a função do sistema de controle gerencial na condução dos negócios.

O ambiente econômico mais competitivo e tecnológico requer uma configuração mais dinâmica dos sistemas de controle gerencial para atender as necessidades organizacionais, com vistas a assegurar a continuidade das operações. Diante dessa realidade, o controle gerencial assume a função de guiar a organizações para enfrentar as novas demandas de gestão, por meio de artefatos que geram medidas qualitativas e quantitativas de desempenho para auxiliar na tomada de decisão.

Como sugestões para futuras pesquisas, considerando as limitações da presente pesquisa, indica-se: a) ampliação do estudo para outras bases de periódicos científicos internacionais e b) realização de estudo similar com periódicos nacionais para comparação de resultados e tendências de assuntos contemporâneos de pesquisa sobre controle gerencial. 


\section{REFERÊNCIAS}

AGUIAR, A. B.; FREZATTI, F. Escolha da estrutura apropriada de um sistema de controle gerencial: uma proposta de análise. Revista de Educação e Pesquisa em Contabilidade, v. I, n. 3, p. 2 I-44, 2007.

ABERNETHY, M. A.; LILLIS, A. M. The impact of manufacturing fexibility on management control system design. Accounting, Organizations and Society, v. 20, n. 4, p. 24I-258, 1995.

ANTHONY, R. N.; GOVINDARAJAN, V. Sistemas de controle gerencial. São Paulo:Atlas, 2006.

ATKINSON, A. A.; BANKER, R. D.; KAPLAN, R. S.; YOUNG, S. M. Contabilidade gerencial. São Paulo:Atlas, 2000.

BARDIN, L. Análise de conteúdo. Lisboa: Edições 70, 1979.

BERRY, A. J.; COAD, A.F.; HARRIS, E.P.; OTLEY, D.T.; STRINGER, C. Emerging themes in management control: a review of recent literature. The British Accounting Review, v. 4I, n. I, p. 2-20, 2009.

BEUREN, I. M.; NASCIMENTO, S. Perfil dos artigos de contabilidade gerencial da Accounting, Organizations and Society publicados de 2005 a 2009. Base - Revista de Administração e Contabilidade da UNISINOS, v. I I, n. 4, p. 332-346, 2014.

BRIZOLLA, M. M. B.; CHIARELLO, T. C.; LAVARDA, C. E. F. Abordagem a respeito da controladoria e contabilidade gerencial: um estudo das redes sociais publicado em periódicos internacionais. Revista de Gestão e Contabilidade da UFPI, v. I, n. I, p. I|9-|40, 2014.

BRUNS, W. J.; WATERHOUSE, J. H. Budgetary control and organization structure. Journal of Ac- counting Research, v. I3, n. 2, p. I77-203, 1975.

CHENHALL, R. H. Management control systems design within its organizational context: findings from contingency-based research and directions for the future. Accounting, Organizations and Society, v. 28, n. 2-3, p. 127-168, 2003.

CUNHA, P. R.; KLANN, R. C.; LAVARDA, C. E. F. Ciclo de vida organizacional e controle gerencial: uma análise dos artigos em periódicos internacionais de contabilidade. Revista de Gestão, Finanças e Contabilidade, v. 3, n. 3, p. 170 I86, 2013.

FARO, M. C. S. C.; SILVA, R. N. S. A natureza da pesquisa em contabilidade gerencial: análise bibliométrica de 1997 a 2007 nos principais periódicos internacionais. In: ENCONTRO DA ASSOCIAÇÃO NACIONAL DE PÓS-GRADUAÇÃO E PESQUISA EM ADMINISTRAÇÃO, 32., 2008, Rio de Janeiro. Anais ... Rio de Janeiro: ANPAD, 2008.

FLAMHOLTZ, E.G. Organizational control systems as a managerial tool. California Management Review, v. 22, n. 2, p. 50-59, 1979. FREZATTI, F.; ROCHA, W.; NASCIMENTO, A. R.; JUNQUEIRA, E. Controle gerencial: uma abordagem da contabilidade gerencial no contexto econômico, comportamental e sociológico. São Paulo: Atlas, 2009.

GALBRAITH, J. R. Designing complex organizations. Reading, MA:Addison-Wesley, 1973.

GOMES, J. S.;AMAT, J. M. Controle de gestão: um enfoque contextual e organizacional. In: CONGRESSO BRASILEIRO DE CUSTOS, 2., 1995, Campinas. Anais ... Campi-
nas:ABC, 1995.

GUERREIRO, R.; FREZATTI, F.; CASADO,T. Em busca de um melhor entendimento da contabilidade gerencial: conceitos da psicologia, cultura organizacional e teoria institucional. Revista Contabilidade \& Finanças, v. 17, n. e., p. 7-2I, 2006.

HENRI, J. F. Management control systems and strategy: a resource-based perspective. Accounting, Organizations and Society, v. 3I, n. 6, p. 529-558, 2006.

HESFORD, J.W.; LEE, S.H.;VAN DER STEDE, W. A.; YOUNG, S. M. Management accounting: a bibliographic study. In: Chapman, C. S.; Hopwood, A. G.; Shields, M. D. (eds.). Handbook of management accounting research. v. I. cap. I. Amsterdam: Elsevier, 2007, p. 3-26.

INTERNATIONAL FEDERATION OF ACCOUNTANTS - IFAC. International Management Accounting Practice Statement I: management accounting concepts. New York: IFAC, 1998.

JENSEN, M. C.; MECKLING, W. H. Theory of the firm: managerial behavior, agency costs and ownership structure. Journal of Financial Economics, v. 3, n. 4, p. 305360, 1976.

KAPLAN, R. S.; NORTON, D. P. The balanced scorecard: translating strategy into action. Boston, MA: Harvard Business School Press, 1996.

KAPLAN, R. S; NORTON, D. A estratégia em ação: balanced scorecard. 7. ed. Rio de Janeiro: Editora Campus, 2000.

LATOUR, B. Reassembling the social: an introduction to the Actor-Network-Theory. Oxford: Oxford University Press, 2005.

LUNKES, R. J.; SCHNORRENBER- 
GER, D. Controladoria: na coordenação dos sistemas de gestão. São Paulo:Atlas, 2009.

MAHAMA, H. Management control systems, cooperation and performance in strategic supply relationships: a survey in the mines. Management Accounting Research, v. I7, n. 3, p. 3I5-339, 2006. MARTINS, G. A.; THEÓPHILO, C. R. Metodologia da investigação científica para ciências sociais aplicadas. 2. ed. São Paulo: Atlas, 2009.

MERCHANT, K. A.; VAN DER STEDE,W.A. Management control systems: performance measurement, evaluation and incentives. England: Pearson Education, 2007. MINTZBERG, $\mathrm{H}$. The structuring of organizations. Englewood Cliffs: Prentice Hall, 1979.

NASCIMENTO,A. R.; RIBEIRO, D. C.; JUNQUEIRA, E. R. Estado da arte da abordagem comportamental da contabilidade gerencial: análise das pesquisas internacionais. In: CONGRESSO USP DE CONTROLADORIA E CONTABILIDADE, 8., 2008, São Paulo. Anais ... São Paulo: USP, 2008.

OUCHI, W. G. A conceptual framework for the design of organizational control mechanisms. Management Science, v. 25, n. 9, p. 833-848, 1979.

OTLEY, D. T. The contingency theory of management accounting: achievement and prognosis. Accounting, Organizations and So- ciety, v. 5, n. 4, p. 4I3-428, 1980.

OTLEY, D. T. Management control in contemporary organizations: towards a wider framework. Management Accounting Research, v. 5, n. 3-4, p. 289-299, 1994.

OTLEY, D.T.; BERRY,A.J. Control, organization and accounting. Accounting, Organization and Society, v. 5, n. 2, p. 23 I-244, 1980.

SHIELDS, M. Research in management accounting by north americans in the 1990s. Journal of Management Accounting Research, v. 9, n. I, p. 3-6I, 1997.

SIMONS, R. Levers of control: how managers use innovative control systems to drive strategic renewal. Boston: Harvard Business School Press, 1995. 